\title{
ESTUDIO COMPARATIVO DE LAS CONDICIONES GENERALES DE LA CONTRATACIÓN DE LAS PRINCIPALES PLATAFORMAS DE REPRODUCCIÓN EN STREAMING: CLÁUSULAS POTENCIALMENTE ABUSIVAS
}

\author{
Aitor Mora Astaburuaga \\ Alumno del MÁster de ACCeso a la Abogacía \\ UNIVERSIDAD DE LA RIOJA \\ Jesús Aitor Prado SEOANE \\ Alumno del Grado en Derecho \\ UNIVERSIDAD DE LA RIOJA
}

SUMARIO: I. Introducción. II. Cláusulas abusivas más comunes. II.I. Establecimiento de limitaciones geográficas. II.2. Libertad del proveedor para modificar tanto los contenidos como el servicio. II.3. Falta de información respecto de los dispositivos compatibles con la plataforma. II.4. Exclusión de responsabilidad en supuestos de calificación incorrecta del contenido. III. Cláusulas abusivas específicas de algunas plataformas de streaming. III.I. Falta de información respecto de los límites de descarga. III.2. Establecimiento de correo electrónico como único medio de facturación. III.3. Establecimiento de un sistema de cancelación de subscripciones perjudicial para el consumidor. III.4. Exclusión de responsabilidad en supuestos de modificación de los dispositivos compatibles sin preaviso. III.5. Obligación al consumidor de eliminar contenidos digitales comprados en caso de terminación discrecional del contrato por parte de la plataforma. III.6. Eliminación discrecional del contenido propiedad del usuario. III.7. Falta de garantías en cuanto a los elementos de conformidad contractual. III.8. Cláusula de renuncia al derecho de desistimiento. IV. Tabla comparativa: condiciones abusivas en las plataformas de streaming. V. Conclusiones. VI. Bibliografía.

RESUMEN: Las nuevas tecnologías han propiciado nuevas formas de entretenimiento y ocio, que resultan cómodas para el consumidor a la vez que lucrativas para quienes las ofrecen. El fenómeno de las plataformas de streaming ya forma parte de nuestras vidas, siendo frecuente la suscripción a estos novedosos servicios; no obstante, dentro del contrato cabe descubrir cláusulas que limitan derechos de consumidores y usuarios, y que deben ser declaradas abusivas. El objetivo de este artículo es analizar -desde un punto de vista práctico apegado al Derecho vivo de los contratos de adhesión- aquellas cláusulas comunes a todas las plataformas de streaming que son susceptibles de ser abusivas, así como el estudio de aquellas cláusulas potencialmente abusivas específicas de alguna plataforma con mayor transcendencia.

Palabras Clave: consumidores; condiciones generales de la contratación; cláusulas abusivas; plataformas streaming

ABSTRACT: New tecnologies have helped to develop new ways of entertainment, that are more comfortable for the consumer and at the same time that they are lucrative for those who offer the service. Streaming platforms phenomenon is already part of our lives, being frequent the subscription to these innovative services, however within the contract we discover clauses that limit the rights of consumers and users, and that must be declared unfair. The goal of this study is to analyze the clauses common to all platforms, verifying their abuse while we pay more attention at those individual clauses that have greater relevance.

KEYWORDS: consumers; standard terms; unfair terms; streaming platforms 


\section{Introducción}

La tecnología streaming (transmisión) ha supuesto desde hace unos años una revolución en el ámbito del entretenimiento doméstico, así como una herramienta muy útil para multitud de empresas, que han hecho de esta tecnología su fuente de riqueza. Tanto es así que no es de extrañar que existan multitud de plataformas que ofrecen servicios en streaming.

La razón por la que las plataformas de streaming se han integrado en nuestra vida cotidiana es porque permiten al consumidor disfrutar de los contenidos digitales sin un horario predeterminado y sin pausas para publicidad (como sí pasa en los medios de telecomunicaciones ordinarios), y, además, con una amplia oferta no solo de contenidos, sino de modos de prestar el servicio, adaptándose así a las necesidades concretas de sus usuarios, existiendo desde plataformas gratuitas hasta plataformas de pago; desde plataformas de suscripción mensual para disfrutar de un amplio catálogo hasta plataformas en las que se alquilan o compran contenidos concretos; desde plataformas que producen sus propios contenidos hasta plataformas en que son los propios usuarios quienes generan el contenido... Sin embargo, este tipo de plataformas tiene algo en común: que están dirigidas exclusivamente a consumidores ${ }^{\mathrm{I}}$.

De este modo, debido a la novedad de este tipo de plataformas es importante realizar un estudio de sus condiciones generales de la contratación (en adelante CGC) en aras de comprobar en qué medida respetan el ordenamiento español en materia de consumo, y, por tanto, en qué medida integran en sus CGC cláusulas abusivas o no, o condiciones que favorezcan situaciones de falta de conformidad ${ }^{2}$ entre lo contratado y el servicio realmente prestado.

Así pues, vamos a proceder a realizar un estudio comparativo de las CGC de las plataformas que hemos considerado más interesantes ${ }^{3}$, que son las de: «Netflix»; «Amazon Prime Video»; «Rakunten TV» ${ }^{4}$; «YouTube»; y «HBO España».

En este sentido, estudiaremos las cláusulas que se encuentran comúnmente en este tipo de condiciones generales, así como una referencia a aquellas que, aun encontrándose solamente en una plataforma, tienen un interés especial.

\footnotetext{
'Encontramos al menos una cláusula en las condiciones generales de la contratación de las plataformas streaming más importantes en las que se establece que el uso será únicamente personal, prohibiéndose cualquier tipo de uso con fines comerciales.

2 Son muy ilustrativos para conocer el régimen general de falta de conformidad tanto actual como el previsible con la entrada en vigor de la Propuesta de Directiva del Parlamento Europeo y del Consejo relativa a ciertos contratos de suministro de contenidos digitales, 9.12.20I5, $\operatorname{COM(2015)634~final~(en~adelante~PDCDig.)~}$ los siguientes artículos: en cuanto al régimen actual CÁMARA LAPUENTE, S., «La nueva protección del consumidor de contenidos digitales tras la Ley 3/20I4, de 27 de marzo», en Revista CESCO de Derecho de Consumo, núm. II, 20I4. En cuanto al régimen que establece la PDCDig., podemos ver CÁMARA LAPUENTE, S., «El régimen de la falta de conformidad en el contrato de suministro de contenidos digitales según la Propuesta de Directiva de 9.I2.20I5», en InDret, núm. 3, 2016 y SPINDLER, G., «Contratos de suministro de contenidos digitales: ámbito de aplicación y visión general de la Propuesta de Directiva de 9.I2.20I5», en InDret, núm. 3, 20 I6.
}

${ }^{3}$ En función de la tipología en la que se integran y su fuerza dentro del mercado español e internacional.

${ }^{4}$ Antes denominada «Wuaki TV», nombre por el que era más conocida. 


\section{Cláusulas abusivas más comunes}

\section{II.I. Establecimiento de limitaciones geográficas ${ }^{5}$}

Hasta hace unos meses esta cláusula no representaba ninguna actividad abusiva, y, de hecho, es una de las cláusulas más frecuentes en las CGC de las plataformas streaming, sin embargo, es necesario apuntar que esta práctica tan frecuente se va a ver, al menos, limitada por la política europea de Mercado Único Digital. De este modo, encontramos que cuando sea aplicable el Reglamento para garantizar la portabilidad Transfronteriza ${ }^{6}$ a partir del 20 de marzo de 2018 se pretende suprimir este tipo de conductas que impiden al consumidor disfrutar de los servicios en un estado miembro que no sea el de su residencia ${ }^{7}$, de modo que devendrán directamente ilícitas al contravenir una norma imperativa, sin necesidad de tener que ponderar su carácter abusivo.

II.2. Libertad del proveedor para modificar tanto los contenidos como el servicio ${ }^{8}$

Es muy frecuente encontrar en las CGC de plataformas de streaming la inserción de una cláusula en la que se otorgue total libertad a la propia plataforma de modificar, sin previo aviso, tanto los contenidos como el propio servicio en sí.

Así, en primer lugar, debemos considerar si la libertad de modificación de contenidos digitales es una cláusula abusiva, para lo que debemos hacernos la siguiente pregunta: ¿es la biblioteca de contenidos contenido esencial del contrato?

Debemos partir de que el contenido esencial del contrato de suministro de contenidos digitales es la puesta a disposición del consumidor de un catálogo (considerablemente amplio) de contenidos de digitales (en las plataformas que hemos seleccionados hablamos tanto de series, como películas y documentales), sin embargo, no podemos considerar, en principio, que sea contenido esencial la presencia de uno o varios contenidos digitales específicos dentro de ese catálogo.

Sin embargo, nos encontramos frecuentemente con campañas publicitarias de las plataformas streaming en las que se destaca la importancia de ciertas series como reclamo publicitario (por ejemplo, la serie «Juego de tronos» para HBO; «Narcos» para Netflix...), por lo que podríamos considerar que, en el caso de que una plataforma suprima de su catálogo dicho contenido publicitado podría considerarse la aplicabilidad del art. 6I TRLGDCU ${ }^{9}$, y, por tanto, la exigencia de integración de la publicidad en la oferta.

\footnotetext{
${ }^{5}$ Cláusula 4.3 CGC de «Netflix»; cláusula 3 CGC de «Amazon Prime Video»; cláusula Condiciones de alta y operatividad del servicio CGC «Rakunten TV»; cláusula 2.I CGC «HBO España».

${ }^{6}$ Reglamento (UE) 20I7/II28 del Parlamento Europeo y del Consejo de I4 de junio de $20 \mathrm{I7}$ relativo a la portabilidad transfronteriza de los servicios de contenidos en línea en el mercado interior.

${ }^{7}$ Vid. considerandos I, 3, 4 y I3.

${ }^{8}$ Cláusula 4.4 CGC de «Netflix»; cláusulas 6.d y 6.e CGC de «Amazon Prime Video»; cláusula Modificación y cancelación del servicio CGC «Rakunten TV»; cláusula 9 CGC «YouTube»; cláusula 2.2 CGC «HBO España».

${ }^{9}$ Real Decreto Legislativo I/2007, de I6 de noviembre, por el que se aprueba el texto refundido de la Ley General para la Defensa de los Consumidores y Usuarios y otras leyes complementarias.
} 
Asimismo, si el consumidor consigue demostrar que la presencia de un determinado contenido en dicha biblioteca fuera la razón de que contratara dicho servicio, y así se lo comunicara al proveedor (y este aceptara), podríamos considerar que existe una falta de conformidad con el contrato si se suprimiera dicho contenido digital del catálogo, en cuanto a que podríamos considerar que dicho contenido dejaría de ser apto para el uso concreto requerido por el consumidor (art. 2.2 b) Directiva 99/44, art. II6 TRLGDCU y art. 6.I b) PDCDig. ${ }^{\text {Io). }}$

Además, es importante destacar el art. I5 PDCDig., pues éste establece un régimen para la modificación de contenidos digitales que permite esta modificación siempre que se den unos supuestos concretos que exigen, al menos, la información suficiente al consumidor de esta modificación con el fin último de que el consumidor, al menos, tenga la opción de cancelar su subscripción sin consecuencias económicas para él ${ }^{\text {II }}$.

Por último, debemos considerar el carácter de estas cláusulas cuando, además de establecer la libre modificación del contenido, también permite al proveedor la modificación del servicio en sí. Entendemos en este caso que será válido siempre que se informe al consumidor de la modificación del servicio de una forma y con una antelación suficiente ${ }^{\mathrm{i}}$.

Sin embargo, en la práctica nos encontramos con cláusulas en las que se establece esta libertad de modificación del servicio sin necesidad de información al consumidor. Es cierto que dichas modificaciones del servicio pueden ser modificaciones accesorias como, por ejemplo, cambiar únicamente el aspecto de la plataforma, pero hay que considerar que la amplitud de esta cláusula abarca la modificación de todo el servicio, esto es, por ejemplo, que una plataforma que preste sus servicios mediante compraventa o alquiler de contenidos digitales y se cambie a un servicio de suscripción; o mejoras que supongan la ralentización del dispositivo utilizado para la reproducción del contenido.

De este modo, debemos considerar que, en la medida en que este tipo de cláusulas, en la práctica, exime al empresario de informar de este tipo de modificaciones pueden generar situaciones de falta de conformidad y, por tanto, el consumidor deberá acudir al sistema establecido en los arts. 65 y 97 TRLGDCU y, en el momento en que se apruebe la PDCDig., a sus arts. I2 a I4.

Asimismo, el art. 85.3 TRLGDCU establece que serán abusivas las cláusulas que «reserven a favor del empresario facultades de interpretación o modificación unilateral del contrato, salvo, en este último caso, que concurran motivos válidos especificados en el contrato», de modo que entendemos que las cláusulas examinadas entrarían, objetivamente, dentro de lo dispuesto en este artículo, pues se dota de libertad al empresario de modificar el contrato (al poder modificarse los contenidos en determinados casos y el propio servicio) sin motivación alguna y sin necesidad de información, no

\footnotetext{
${ }^{10}$ Propuesta de Directiva del Parlamento Europeo y del Consejo relativa a determinados aspectos de los contratos de suministro de contenidos digitales, de 9.I2.20I5, COM(20I5) 634 final.

${ }^{\text {" }}$ Cabe destacar que este artículo, como la generalidad de la Propuesta de Directiva, subordina esta posibilidad a la voluntad del empresario, pues el primer supuesto de libre modificación de contenidos digitales es la inserción en el contrato de una cláusula en la que se prevea esta posibilidad.

${ }^{12}$ Entendemos como información suficiente en tiempo y forma aquella que contenga, de una forma comprensible, la amplitud de los cambios que se van a producir en el servicio, y que se haga con una antelación de al menos 30 días para que el consumidor tenga tiempo suficiente para considerar si desistir del contrato o no.
} 
estableciéndose en las propias condiciones generales causas tasadas de modificación contractual.

II.3. Falta de información respecto de los dispositivos compatibles con la plataforma ${ }^{\mathrm{I3}}$

Es también muy habitual encontrar en estas plataformas cláusulas que contravienen todo tipo de obligación de información precontractual, pues, a pesar de la obligación que tienen de especificar en sus condiciones generales cuál es el software necesario para poder utilizar la plataforma o, incluso, cuáles son los dispositivos compatibles con dicha plataforma, lo que conlleva una infracción del deber de información del proveedor en cuanto a la interoperabilidad y la funcionalidad ${ }^{14}$ de las plataformas ${ }^{15}$ establecido en los arts. 6o y 97 TRLGDCU.

Asimismo, es destacable que existen plataformas que, para dar apariencia de que sí informan al consumidor de todo lo relativo a interoperabilidad y funcionalidad de la plataforma insertan links en las CGC que remiten a otras páginas web que informan de los requisitos necesarios que debe cumplir el dispositivo del consumidor para poder acceder a los contenidos digitales prestados por las plataformas streaming. Sin embargo, en la práctica, las páginas a las que remiten estos enlaces no presentan la totalidad de la información necesaria para conocer los requisitos de funcionalidad e interoperabilidad, teniendo que acceder a varias páginas web distintas para acceder a esta información.

De este modo, podría aplicarse cierta falta de transparencia en cuanto a la información precontractual al no constar tal información donde debería, lo que conllevaría una infracción de los deberes de información establecidos en los artículos 6o.r.i, y 97.I. s) y t) TRLGDCU, que puede derivar, además, en una falta de conformidad ex art. II6 TRLGDCU.

También es importante destacar que algunas de estas plataformas ni siquiera establecen un enlace operativo para conocer esta información, por lo que incumplirían este deber de información precontractual en todo caso.

${ }^{13}$ Cláusula 4.8 CGC de «Netflix»; cláusula 2 CGC de «Amazon Prime Video»; cláusula Utilización del Servicio a través de las aplicaciones disponibles para los dispositivos autorizados CGC «Rakunten TV».

${ }^{14}$ Estos requisitos se encuentran recogidos en el art. 6o TRLGDCU, de modo que nos sirve para conceptualizar su alcance en CÁmARA LAPUENTE, S., «La nueva protección ...», op. cit. Así, la funcionalidad se define como: «las posibles maneras de utilizar el contenido digital» (Cdo. I9 Directiva 99/44), lo que se ve completado por el art. 60.2 i) TRLGDCU al incluir al concepto «las medidas técnicas de protección aplicables, como son, entre otras, la protección a través de la gestión de los derechos digitales o la codificación regional». Por otro lado, el mismo considerando de la Directiva, como los arts. 6o y 97 TRLGDCU establecen la interoperabilidad como «programas estándar» que sean compatibles con el contenido digital y que, como apunta CÁMARA LAPUENTE en el artículo que hemos citado, sean «conocidos por el empresario o que quepa esperar razonablemente que conozca».

${ }^{15}$ Debería exponer si necesita Adobe Flash player io por ejemplo, u otros softwares licenciados a los cuales el usuario debe tener acceso para disfrutar del contenido digital. 
II.4. Exclusión de responsabilidad en supuestos de calificación incorrecta del contenido $^{16}$

Hemos encontrado también, en varias de estas plataformas, una cláusula que exonera al empresario de cualquier tipo de responsabilidad derivada de la incorrecta clasificación de estos contenidos. Sin embargo, entendemos que, en la medida en que este tipo de errores de clasificación pueden generar situaciones perjudiciales para el consumidor $^{17}$ no podrá eximirse totalmente de su responsabilidad y, por tanto, debemos considerar la abusividad de dicha cláusula ex art. 86.I TRLGDCU.

\section{Cláusulas abusivas específicas de algunas plataformas de streaming}

\section{III.I. Falta de información respecto de los límites de descarga ${ }^{\text {18 }}$}

Nos encontramos en este caso con una cláusula específica de la plataforma «Netflix» en la que se establece que existe un límite para descargar (siempre dentro de la propia plataforma) contenidos digitales. Esta cláusula incumpliría en cierta medida las obligaciones precontractuales previas del articulo 60 y 97.I s) y t) del TRLGDCU, en los que se estipula los deberes de información relativos a la funcionalidad e interoperabilidad. En este caso «Netflix» no especifica ni el número de títulos que corresponden por dispositivo (ni máximo ni mínimo), ni tampoco establece la cantidad de datos máxima que se puede descargar, por lo que la información que presta resulta insuficiente para que el consumidor conozca las características del contrato $^{\text {I9 }}$, y cabría que el usuario planteara una falta de conformidad con el contrato.

En cuanto a la aplicación de la PDCDig. se refiere, debemos atender que nos encontramos con un supuesto en el que deberíamos aplicar el parámetro objetivo de conformidad contenido en el art. 6.2 PDCDig. ${ }^{20}$, pues, como vemos, se incumple con el criterio de transparencia que debe tener el clausulado, ya que, aunque el texto sea claramente comprensible y sea fácil deducir que existen limitaciones a las descargas de

\footnotetext{
${ }^{16}$ Cláusula 6.b CGC «Amazon Prime Video»; cláusula LIMITACIÓN Y/O EXCLUSIÓN DE GARANTÍAS Y DE RESPONSABILIDAD CGC «Rakunten TV»; cláusula 7.9 CGC «YouTube».

${ }^{17}$ v.gr: Puede generar ciertos trastornos en un menor de edad el visionado de ciertos contenidos que no han sido clasificados, o habiendo sido clasificados defectuosamente resultan dañinos para el público no autorizado. Por ejemplo, si una película «Kill Bill» está calificada como película infantil y un padre decide ver esa película en compañía de su hijo, sin saber realmente el género de la misma, dicha calificación defectuosa ocasionará un perjuicio sobre el consumidor (trastornos en la mente del niño), por lo que la plataforma no podrá exonerarse de todo tipo de responsabilidad por un contenido que debería estar clasificado correctamente.

${ }^{18}$ Cláusula 4.5 CGC «Netflix».

19 No especifica un número máximo de contenidos que pueden descargarse, ni una cantidad de datos; tampoco se especifica si dicho límite de descarga es periódico (ya sea mensual, a razón de x descargas por mes, o semana, por ejemplo).

${ }^{20}$ Art. 6.2 PDCDig.: «En el supuesto de que el contrato no establezca, cuando proceda, de forma clara y comprensible, los requisitos para los contenidos digitales de conformidad con el apartado I, estos serán aptos para los fines a los que ordinariamente se destinen contenidos digitales del mismo tipo, incluida su funcionalidad, interoperabilidad y demás características de funcionamiento tales como la accesibilidad, la continuidad y la seguridad (...)».
} 
contenidos para su visualización offline, no se establece en ningún momento cuáles son dichas limitaciones, y ni siquiera remite a otro texto o enlace para indicar cuáles son estas limitaciones, de modo que, aunque sea fácilmente comprensible dicha cláusula no es transparente en el sentido de que falta la información más relevante, cuáles son concretamente dichos límites.

Además, dicha falta de transparencia afecta a la funcionalidad del servicio, pues la funcionalidad «se refiere a las posibles formas de utilización del contenido digital», así como a la «ausencia o presencia de restricciones técnicas» y en este supuesto nos encontramos con que, al omitir el listado de limitaciones, se está afectando a la forma de utilización del bien.

De este modo, entendemos que nos encontraremos, una vez entre en vigor la propuesta de directiva, con una falta de conformidad objetiva en relación con lo dispuesto en el art. 6.2 PDCDig.

\section{III.2. Establecimiento de correo electrónico como único medio de facturación ${ }^{2 \mathrm{I}}$}

Nos encontramos nuevamente con una cláusula que establece «Netflix» en sus CGC. En esta cláusula la plataforma determina como único medio de comunicación de las facturas el correo electrónico cuando el propio TRLGDCU establece el derecho del consumidor de recibir dicha documentación en formato papel. De este modo, debemos sostener que esta cláusula vulnera lo dispuesto en el art. 63.3 TRLGDCU pues el empresario deberá obtener consentimiento expreso del consumidor para enviar la factura vía electrónica cuando aquí no se está solicitando dicho consentimiento, sino que se establece directamente en las condiciones generales como forma única de expedir la factura.

III.3. Establecimiento de un sistema de cancelación de subscripciones perjudicial para el consumidor ${ }^{22}$

En este caso no estamos hablando de una única cláusula, sino que nos referimos al estudio conjunto de dos cláusulas de la plataforma «Amazon Prime Video» que establecen un régimen que perjudica gravemente los derechos del consumidor.

En estas dos cláusulas observamos, cómo se establece por una parte la facultad de modificación unilateral por el propio proveedor de los contenidos esenciales del contrato; y por otra la facultad de cancelación de la suscripción (esto es, la resolución del contrato) por parte del consumidor de forma unilateral (con algunos matices).

Si contemplamos estas cláusulas de forma general podrían ser válidas, pues, en caso de que el proveedor modifique las condiciones esenciales del contrato (tales como el precio), si el consumidor no está de acuerdo con estos cambios podrá resolver el contrato de forma unilateral y sin necesidad de justificación. Sin embargo, vemos cómo en las CGC de esta plataforma se permite al proveedor hacer uso de esta facultad sin necesidad alguna de notificación, lo que hace que, a efectos reales, el consumidor pueda verse perjudicado por

\footnotetext{
${ }^{21}$ Cláusula 3 CGC «Netflix».

${ }^{22}$ Cláusulas 4.c y 4.d CGC «Amazon Prime Video».
} 
estas modificaciones unilaterales sin poder hacer efectivo su derecho de resolución del contrato en el momento oportuno. No es difícil imaginar situaciones en las que el proveedor modifique las condiciones del contrato sin previo aviso (que aumente el precio y reduzca las prestaciones del servicio, por ejemplo) y debido a esta falta de notificación el consumidor siga utilizando el servicio sin darse cuenta, en un primer momento, de que éstas han cambiado y que posteriormente vea la factura, por ejemplo, y se percate de esta subida de precio y de la reducción de las prestaciones. En estas situaciones, el consumidor, al haber utilizado el servicio, deberá realizar el pago de esa mensualidad, aunque desee resolver el contrato, viéndose de este modo perjudicado.

Si se estableciera en dicha cláusula un deber de información por parte del proveedor de estas modificaciones con un plazo suficiente para que el consumidor pueda ejercer efectivamente la cancelación de su suscripción sin verse perjudicado podríamos considerar que las cláusulas fueran válidas.

Por lo tanto, podríamos considerarla como abusiva ex artículo 82 TRLGDCU, debido a un desequilibrio importante en los derechos y obligaciones de las partes ya que, como hemos visto, estas cláusulas dejan al consumidor en una situación en la que, aun pudiéndose generar una situación de disconformidad con el contrato (en cuanto a sus características principales) éste deberá realizar el pago de al menos una mensualidad más.

Asimismo, también consideramos la abusividad de esta cláusula en relación con el art. 85 TRLGDCU, pues realmente se estaría dejando a la voluntad del empresario la libre modificación del contrato.

III.4. Exclusión de responsabilidad en supuestos de modificación de los dispositivos compatibles sin preaviso $^{23}$

Nos encontramos en este caso con una cláusula de la plataforma «Rakunten TV» en la que se establece la exención de toda responsabilidad de la plataforma en los casos en los que se modifique la lista de dispositivos compatibles sin necesidad de informar de dichos cambios al consumidor. De este modo, entendemos que esta cláusula genera una situación en la que el consumidor se puede ver privado de su capacidad de disponer del servicio contratado porque de un momento a otro la plataforma haya decidido dejar de dar soporte al dispositivo que el consumidor utilizaba para disfrutar del contenido de la plataforma. Es cierto que con los avances tecnológicos y, por tanto, las sucesivas actualizaciones de los sistemas operativos de los distintos dispositivos que hay en el mercado hace imposible que la plataforma siga dando soporte a determinados dispositivos cuya tecnología haya devenido obsoleta. Sin embargo, lo que hace cuestionable a esta cláusula es la exención de responsabilidad en caso de no informar de esta decisión al consumidor, el cual se puede ver directamente afectado al seguir pagando por el servicio cuando realmente no puede disfrutarlo y, por tanto, se generaría una situación de falta de conformidad contractual; además de que podríamos considerar en estos casos la falta de causa sobrevenida del

${ }^{23}$ Cláusula Uso de dispositivos autorizados CGC «Rakunten TV». 
contrato al romperse el equilibrio característico de las obligaciones sinalagmáticas ex art. II24 CC.

III.5. Obligación al consumidor de eliminar contenidos digitales comprados en caso de terminación discrecional del contrato por parte de la plataforma ${ }^{24}$

Nos encontramos con otra cláusula de la plataforma «Rakunten TV». En este caso, la empresa establece en las CGC la obligación del consumidor de eliminar todos aquellos contenidos digitales que haya comprado y descargado en caso de que la plataforma decida resolver, de forma discrecional, el contrato que le vincula con el consumidor.

En primer lugar, la plataforma califica su servicio como una compraventa en su cláusula «Descripción del Servicio» al establecer que «El Servicio de Rakunten TV incluye, a título enunciativo: Alquiler, venta o difusión gratuita de los Contenidos Digitales (...)».

De este modo, como la propia plataforma califica el negocio jurídico como compraventa serán de aplicación los arts. I462 y ss. CC, de modo que habrá una transmisión de la propiedad (con algunas limitaciones en cuanto al uso de los contenidos digitales derivadas de los derechos de propiedad intelectual). Sin embargo, la plataforma trata de obligar al consumidor a eliminar dicho contenido adquirido mediante compraventa por el mero hecho de terminar la relación contractual existente entre ambos, lo que va en contra de la propia naturaleza de este contrato.

Por este motivo, entendemos que esta cláusula cabría en el tipo contemplado en el art. 86 TRLGDC, pues se estaría privando al consumidor de sus derechos de propiedad sin justificación alguna.

\section{III.6. Eliminación discrecional del contenido propiedad del usuario ${ }^{25}$}

Esta cláusula puede ser abusiva para el usuario que sube un contenido a una plataforma de streaming, cuando dicho contenido sea de su propiedad exclusiva y no haya cedido ningún derecho de propiedad ni total ni parcial a favor de la plataforma. No obstante, en el caso de «YouTube», -quien explota el contenido que se encuentra en su sitio web, como licenciataria de usuarios y creadores de contenido- no informa correctamente al usuario que dicha cesión, lleva aparejada la cesión de ciertos derechos para con el contenido, siendo una de ellas la relativa a su eliminación discrecional de la propia plataforma.

Asimismo, podremos considerar dicha cláusula como abusiva por no respetar el deber de transparencia y por la ausencia de información precontractual; apreciamos una manifiesta vinculación a la voluntad del empresario en tanto en cuanto al redactar dicha cláusula no se especifica de forma correcta las consecuencias que dicha cesión produce en los derechos del usuario ex artículo 82.4. a y b TRLGDCU. En cuanto a la ausencia de información precontractual -el cual es un deber impuesto al empresario, y que, llegado el momento de no cumplir con dicho deber, las consecuencias poseen una especial

\footnotetext{
${ }^{24}$ Cláusula Incumplimiento CGC «Rakunten TV».

${ }^{25}$ Cláusula 7.8 CGC «YouTube».
} 
relevancia- se recoge ex artículos 97.I. TRLGDCU, y estipula que «el empresario deberá facilitarle de forma clara y comprensible, salvo que resulte manifiesta por el contexto, la información relevante, veraz y suficiente sobre las características principales del contrato, en particular sobre sus condiciones jurídicas y económicas». Por ello podemos entender que supone una falta de información el hecho de que no se expliquen las consecuencias jurídicas que conlleva la cesión de derechos de propiedad a la hora de «subir» a la plataforma el contenido deseado.

Por último, cabría plantearse si el empresario debido a la cesión antes mencionada está disponiendo de un derecho que le permite retirar ese contenido de forma unilateral determinando de esta forma si el bien o servicio se adecua a lo estipulado en el contrato, lo que vulneraría también lo dispuesto en el artículo 85.II TRLGDCU por una vinculación manifiesta del contrato a la voluntad del empresario.

III.7. Falta de garantías en cuanto a los elementos de conformidad contractual ${ }^{26}$

Establece «YouTube» en esta cláusula que no garantizará en ningún caso que el servicio prestado se lleve a cabo sea seguro, que se realice sin error, o de forma ininterrumpida; tampoco garantiza que cumpla con las expectativas del consumidor y ni siquiera garantiza que se corrijan los defectos relativos a la funcionalidad e interoperabilidad del servicio. De este modo, entendemos que realmente esta cláusula exime de responsabilidad a la plataforma en cuanto no cumpla con los requisitos de conformidad contractual y, por este motivo, incumple con los requisitos establecidos entre otros artículos en el 97 TRLGDCU.

Por este motivo, consideramos que se está incurriendo mediante esta cláusula en lo dispuesto en el art. 86.I TRLGDCU, el cual se refiere a «cláusulas que modifiquen, en perjuicio del consumidor y usuario, las normas legales sobre conformidad con el contrato», y, por consiguiente, esta cláusula debe ser considerada abusiva.

III.8. Cláusula de renuncia al derecho de desistimiento ${ }^{27}$

El derecho de desistimiento se funda en la ausencia de examen por parte del consumidor de aquello que pretende adquirir, y por ello se le permite un plazo ampliado para que si lo que adquirido o contratado no cumple con sus expectativas pueda desistir del contrato. Se regula en el artículo IO4 a) y b) TRLGDCU, posibilitando un plazo de desistimiento de I4 días desde la celebración del contrato hasta la ejecución del mismo ${ }^{28}$.

\footnotetext{
${ }^{26}$ Cláusula I2 CGC «YouTube».

${ }^{27}$ Cláusula I2 CGC «HBO España».

${ }^{28}$ Véanse: SoSA OlÁN, H., «Capítulo II. Naturaleza jurídica del derecho de desistimiento en los contratos celebrados con consumidores», en El derecho de desistimiento como mecanismo protector del consumidor en la contratación electrónica, Salamanca, Ediciones Universidad de Salamanca, 20I5, págs. I6I-I84; y GARCíA GARCíA, L. M. ${ }^{\text {, }}$ «Régimen general del derecho de desistimiento», en DE LEÓN ARCE, A. (Dir.), Derechos de los consumidores

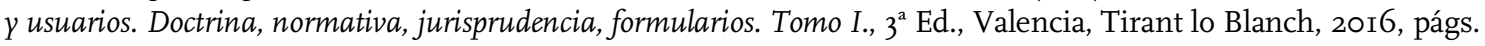
559-60I.
} 
«HBO España» pretende con esta cláusula que el consumidor renuncie a dicho derecho desde el mismo momento en que se celebra el contrato, negándole el derecho a desistir en los siguientes i4 días naturales. De este modo, la cláusula de la que estamos hablando trata de informar al consumidor de que en este contrato no le asiste el derecho al desistimiento.

Es cierto que el art. I03 TRLGDCU establece una serie de situaciones en las que se puede privar al consumidor de este derecho. En concreto, el art. I03 m) TRLGDCU establece la posibilidad de que el consumidor pierda este derecho «cuando la ejecución haya comenzado con el previo consentimiento expreso del consumidor y usuario».

Asimismo, cabe preguntarse por cuándo comienza la ejecución del contrato. En aquellas plataformas de streaming que se basen en el método de subscripción -como «Netflix» O «HBO España», donde el consumidor disfruta de un servicio de reproducción en video en streaming- podemos plantear si el mero acceso a la plataforma resulta en sí mismo una ejecución del contrato, sin necesidad de que se reproduzca ningún contenido. Esta opción no parece, a priori, ser la más acertada debido a la naturaleza del servicio que se presta, pues la plataforma es un mero medio para disfrutar del verdadero servicio, la reproducción de vídeo en streaming, de modo que cabría considerar que la verdadera ejecución del contrato comenzaría en el momento en que se reproduzca algún contenido digital por primera vez.

Por otro lado, no resulta concebible que al aceptar las condiciones generales de la contratación que propone «HBO España», el propio consumidor este manifestando un «consentimiento reforzado» ${ }^{29}$ del que se pueda desprender que la ejecución del contrato ha comenzado, y en consecuencia que el consumidor haya renunciado a su derecho a desistir.

Debido a que la norma exige que dicho «consentimiento reforzado» se recabe de forma expresa -como marcando una casilla, por ejemplo-, pero en ningún caso mediante el uso de casillas marcadas previamente por el empresario, ni siquiera con la inserción de una cláusula dentro de las condiciones generales que una vez aceptadas en bloque puedan derivar en que dicho consentimiento reforzado haya sido recabado correctamente, como entendemos que es el caso. También se exige una confirmación emitida por parte del empresario de que dicho «consentimiento reforzado» ha sido recabado y que por ende se inicia la ejecución del contrato.

«HBO España» no recaba correctamente el «consentimiento reforzado» ni emite confirmación de dicho consentimiento, por ende, no puede ampararse en la excepción dispuesta en el artículo I03 m) TRLGDCU y, por lo tanto, el derecho de desistimiento sí resultaría aplicable, incluso en el caso de que el contrato haya comenzado su ejecución ${ }^{30}$.

En conclusión, dicha cláusula es considerada abusiva por dos vías: en primer lugar, porque limita los derechos de consumidores y usuarios ya que restringe el ejercicio del derecho de desistimiento al imponerle su renuncia a la hora de la celebración del contrato

\footnotetext{
${ }^{29}$ Para una mayor profundización en el derecho de desistimiento en el contrato sobre contenidos digitales consultar CÁmARA LAPUENTE, S., «La nueva protección ...», op. cit, pp.I54.

${ }^{30}$ Dicho incumplimiento tanto formal como material le acarrea al empresario diferentes sanciones civiles contempladas en el artículo I08.4 TRLGDCU, tales como la exención por parte del consumidor de cualquier tipo de coste a la hora de ejercer el desistimiento. CÁmARA LAPUENTE, S., «La nueva protección ...», op. cit,
} 
(sin cumplir con los criterios establecidos en el art. I03 TRLGDCU) -vía artículo 82.4 b) TRLGDCU-; y, en segundo lugar, porque priva al consumidor de derechos reconocidos por una norma dispositiva o imperativa vía artículo 86.I y 86.7 TRLGDCU más concretamente en lo relativo a limitar los derechos básicos del consumidor o usuario.

\section{Tabla comparativa: condiciones abusivas en las plataformas de streaming}

Por último, nos parece interesante incluir en este estudio comparativo un cuadro ilustrativo en el que se muestra qué cláusulas potencialmente abusivas contiene cada una de las principales plataformas de reproducción de vídeo en streaming según la normativa española. ${ }^{3+}$

\begin{tabular}{|c|c|c|c|c|c|}
\hline & NETFLIX & $\begin{array}{l}\text { AMAZON } \\
\text { PRIME }\end{array}$ & $\begin{array}{l}\text { RAKUNTEN } \\
\text { TV }\end{array}$ & YOUTUBE & $\begin{array}{l}\text { HBO } \\
\text { ESPAÑA }\end{array}$ \\
\hline Limitación geográfica & $\checkmark$ & $\checkmark$ & $\checkmark$ & $x$ & $\checkmark$ \\
\hline $\begin{array}{l}\text { Libre modificación de } \\
\text { contenido/servicios }\end{array}$ & $\checkmark$ & $\checkmark$ & $\checkmark$ & $\checkmark$ & $\checkmark$ \\
\hline $\begin{array}{l}\text { No especificación de } \\
\text { dispositivos compatibles }\end{array}$ & $\checkmark$ & $\checkmark$ & $\checkmark$ & $x$ & $x$ \\
\hline $\begin{array}{l}\text { Exclusión de } \\
\text { responsabilidad: no } \\
\text { calificación correcta del } \\
\text { contenido }\end{array}$ & $x$ & $\checkmark$ & $\checkmark$ & $\checkmark$ & $x$ \\
\hline $\begin{array}{l}\text { No especificación de } \\
\text { límites de descarga }\end{array}$ & $\checkmark$ & $x$ & $x$ & $x$ & $x$ \\
\hline $\begin{array}{l}\text { Único medio de } \\
\text { facturación electrónico }\end{array}$ & $\checkmark$ & $x$ & $x$ & $x$ & $x$ \\
\hline $\begin{array}{l}\text { Sistema de cancelación } \\
\text { de subscripciones } \\
\text { perjudicial para el } \\
\text { consumidor }\end{array}$ & $x$ & $\checkmark$ & $x$ & $x$ & $x$ \\
\hline $\begin{array}{l}\text { Exclusión de } \\
\text { responsabilidad: } \\
\text { modificación de } \\
\text { dispositivos compatibles } \\
\text { sin preaviso }\end{array}$ & $x$ & $x$ & $\checkmark$ & $x$ & $x$ \\
\hline $\begin{array}{l}\text { Terminación } \\
\text { discrecional del contrato } \\
\text { obligando al consumidor } \\
\text { a eliminar contenido } \\
\text { comprado }\end{array}$ & $x$ & $x$ & $\checkmark$ & $x$ & $x$ \\
\hline
\end{tabular}

${ }^{31}$ El símbolo $\checkmark$ significa que la plataforma correspondiente contiene dicha cláusula inserta en sus CGC. El símbolo $\times$ significa que la plataforma correspondiente en el cuadro no contiene dicha condición inserta en sus CGC. 


\begin{tabular}{l|c|c|c|c|c}
$\begin{array}{l}\text { Eliminación discrecional } \\
\text { del contenido del usuario }\end{array}$ & $x$ & $x$ & $x$ & $\checkmark$ & $x$ \\
\hline $\begin{array}{l}\text { Falta de garantías en } \\
\text { cuanto a elementos de } \\
\text { conformidad }\end{array}$ & $x$ & $x$ & $x$ & $\checkmark$ & $x$ \\
\hline $\begin{array}{l}\text { Renuncia al derecho de } \\
\text { desistimiento }\end{array}$ & $x$ & $x$ & $x$ & $x$ & $\checkmark$ \\
\hline
\end{tabular}

Fuente de la tabla: elaboración propia a partir de las CGC de las distintas plataformas estudiadas

\section{Conclusiones}

De la elaboración de este estudio comparativo cabe destacar las siguientes conclusiones:

I. En primer lugar, todas las condiciones generales de la contratación que hemos estudiado contienen una gran variedad de cláusulas que podrían ser consideradas abusivas, por lo que debemos considerar que cabe un amplio margen de mejora en lo que la elaboración legal de dichas condiciones se refiere.

2. En segundo lugar, que la plataforma (de las estudiadas) que contiene un menor número de cláusulas abusivas es «HBO España», por lo que consideramos que ésta es la plataforma que otorga mejores condiciones jurídicas al consumidor (sin entrar a considerar el catálogo de contenidos digitales).

3. En tercer lugar, que la plataforma que contiene más cláusulas abusivas es «Rakunten TV», y, por consiguiente, la plataforma que peores condiciones jurídicas otorga al consumidor.

4. En cuarto lugar, todas las plataformas streaming estudiadas contienen al menos una cláusula de exención de responsabilidades y absolutamente todas se confieren a sí mismas la capacidad de modificar los contenidos digitales y/o el servicio en sí mismo. Asimismo, hemos observado la gran frecuencia en las distintas plataformas de cláusulas que contravenían el deber de información precontractual de los consumidores, en particular, en lo relativo a su funcionalidad e interoperabilidad.

5. Por último, es preciso apuntar que, a pesar de que hemos destacado algunas de las cláusulas abusivas que hemos considerado más frecuentes o con mayor impacto en los derechos de los consumidores, las cláusulas abordadas en este estudio no son las únicas que pueden contener carácter abusivo. Sin embargo, debemos matizar que las cláusulas abusivas representan un pequeño porcentaje dentro de las CGC de las plataformas de streaming. 


\section{Bibliografía}

Bercovitz Rodríguez Cano, R. (Cord.), Comentario del texto refundido de la Ley general para la defensa de los consumidores $y$ usuarios $y$ otras leyes complementarias, $2^{a}$ Ed., Thomson Reuters Aranzadi, Pamplona, 2015.

CÁmara Lapuente, S. (Cord.), Comentarios a las normas de protección de los consumidores, Madrid, Colex, 20II.

CÁmARA LAPUENTE, S., «La nueva protección del consumidor de contenidos digitales tras la Ley 3/20I4, de 27 de marzo», en Revista CESCO de Derecho de Consumo, núm. II, 2014.

CÁmARA LAPUente, S., «El régimen de la falta de conformidad en el contrato de suministro de contenidos digitales según la Propuesta de Directiva de 9.I2.20I5», en InDret, 3, Barcelona, 2016.

DE LeÓn ARCE, A. (Dir.), Derechos de los consumidores y usuarios. Doctrina, normativa, jurisprudencia, formularios. Tomo I., $3^{\mathrm{a}}$ Ed., Tirant lo Blanch, Valencia, 2016.

Sosa Olán, H., El derecho de desistimiento como mecanismo protector del consumidor en la contratación electrónica, Salamanca, Ediciones Universidad de Salamanca, 2015.

SPINDLER, G., «Contratos de suministro de contenidos digitales: ámbito de aplicación y visión general de la Propuesta de Directiva de 9.I2.2015», en InDret, núm. 3, 2016.

\section{Condiciones generales de la contratación}

- Condiciones Generales de la Contratación de «Amazon Prime Video»

(vid en: https://www.amazon.es/gp/help/customer/display.html?nodeId=200545940; fecha de consulta: II.II.2OI7).

- Condiciones Generales de la Contratación de «HBO España» (vid en: https://es.hboespana.com/account/terms; fecha de consulta: II.II.2OI7).

- Condiciones Generales de la Contratación de «Netflix» (vid en: https://help.netflix.com/legal/termsofuse; fecha de consulta: II.II.20I7).

- Condiciones Generales de la Contratación de «Rakunten TV» (vid en: https://es.rakuten.tv/terms_conditions/373; fecha de consulta: II.II.20I7).

- Condiciones Generales de la Contratación de «YouTube» (vid en: https://www.youtube.com/static?gl=ES\&template=terms\&hl=es; fecha de consulta: II.II.2OI7).

\section{Legislación considerada}

- Propuesta de Directiva del Parlamento Europeo y del Consejo relativa a determinados aspectos de los contratos de suministro de contenidos digitales, de 9.12.20I5, COM (2015) 634 final.

- Real Decreto Legislativo I/2007, de I6 de noviembre, por el que se aprueba el texto refundido de la Ley General para la Defensa de los Consumidores y Usuarios y otras leyes complementarias.

- Reglamento (UE) 20I7/II28 del Parlamento Europeo y del Consejo relativo a la portabilidad transfronteriza de los servicios de contenidos en línea en el mercado interior, de i4.7.20I7. 